\title{
Aspectos psicológicos de la supervivencia en operaciones militares
}

\author{
Martínez Sánchez JA. ${ }^{1}$
}

Sanid. mil. 2011; 67 (1): 43-48; ISSN: 1887-8571

\begin{abstract}
RESUMEN
El adiestramiento y entrenamiento militar en supervivencia enfatiza la preparación física y la adquisición de determinados conocimientos técnicos, concediendo escasa relevancia a la preparación psicológica. Partiendo de este hecho, en el presente artículo se exponen y analizan brevemente una serie de factores psicológicos que condicionan y limitan la adaptación del militar que se ve inmerso en una situación de supervivencia. Seguidamente se presentan una serie de pautas y medidas psicológicas que, incidiendo en los aspectos anteriormente señalados, tienen como objetivo mejorar y favorecer la adaptación del militar a dichas situaciones, incrementando de esa manera sus posibilidades de superarlas con éxito.
\end{abstract}

PALABRAS CLAVE: Supervivencia, Entrenamiento, Aspectos psicológicos, Estresores, Actitud.

\section{Psychological aspects of survival in military operations \\ SUMMARY}

Military survival training emphasizes physical fitness and the acquisition of technical skills, giving little importance to psychological preparation. Given this, this article presents and briefly analyzes a number of psychological factors that influence and limit the adaptation of the military member who is engaged in a survival situation. It then presents a set of guidelines and psychological measures, affecting the aspects described above, which are intended to improve and promote the adaptation of the military member to such situations, thereby increasing their chances of successfully overcoming them.

KEY WORDS: Survival, Training, Psychological factors, Stressors, Attitude.

\section{INTRODUCCIÓN}

Entendemos por situación de supervivencia aquella en la que un individuo se encuentra inmerso en un medio generalmente desconocido y adverso ${ }^{1}$, en el que deberá subsistir con los recursos que le proporciona el terreno, manteniendo las condiciones psicofísicas necesarias para sobrevivir ${ }^{2}$. Un accidente aéreo, un amerizaje en el mar, quedarse aislado durante la ejecución de una misión o ejercicio militar son situaciones probables en la vida de cualquier militar. Por tanto, todo militar necesita aprender supervivencia para poder afrontar ese tipo de situaciones con posibilidades de éxito.

La mayoría de cursos, libros y manuales que enseñan supervivencia se centran en cuestiones técnicas, prestando poca atención a los aspectos psicológicos. Valga como ejemplo de ello que, si bien ambos señalan la importancia de dichos aspectos, tanto el FM 21$76^{3}$-manual de supervivencia del Ejército estadounidense- como su homónimo español el Manual de Enseñanza ME6-003², apenas dedican un capítulo a la psicología de la supervivencia. Más curioso es el caso del Manual de Instrucción sobre Supervivencia del Ejército español, M17-0024, que ni siquiera menciona estos aspectos.

Sin embargo, para afrontar con éxito una situación de supervivencia no solo se requiere unos conocimientos técnicos, sino que es

${ }^{1}$ Cap. Psicólogo. Academia General del Aire.

Dirección para correspondencia: Academia General del Aire. Jefatura de Estudios. 30720 Santiago de la Ribera. Murcia. E-mail: jamartsan@ea.mde.es

Recibido: 27 de enero de 2010

Aceptado: 29 de marzo de 2010 necesario también poseer y entrenar ciertas habilidades mentales o psicológicas. En efecto, en supervivencia hay dos grandes aspectos a considerar: el técnico y el psicológico. El aspecto técnico aparece en todos los manuales de supervivencia y se refiere a un conjunto de conocimientos y técnicas necesarias para sobrevivir: construcción de refugios, obtención de agua y alimentos, medios de orientación y posicionamiento, etc. La preparación técnica es necesaria, pero no suficiente para salir airoso de una situación de supervivencia. Un ingrediente clave en toda situación de supervivencia es la actitud del superviviente ${ }^{3}$, como lo es aceptar inmediatamente la realidad de la nueva situación y reaccionar de manera adecuada 5 . De hecho, personas bien entrenadas han muerto por perder la calma y no ser capaz de poner en práctica dicho entrenamiento. Por tanto, se necesita una preparación psicológica adecuada, basada en una actitud positiva del individuo y en su deseo de sobrevivir.

Es precisamente para sobrevivir que el superviviente deberá afrontar y superar una serie de retos u obstáculos que en última instancia pueden terminar mermando su moral. Miedo, frío, frustración, hambre, sed, cansancio, dolor, soledad... son algunos de ellos. Estos obstáculos generan pensamientos y emociones que pueden convertir a un militar bien entrenado y seguro de sí mismo en un individuo indeciso e ineficaz, cuya capacidad para sobrevivir se va a encontrar notablemente disminuida. Por tanto, todo superviviente debe ser capaz de reconocer la gran variedad de tensiones a las que se verá sometido, así como de controlar sus sentimientos y reacciones ante ellas.

En consecuencia, un entrenamiento adecuado en supervivencia debe abarcar tanto la preparación técnica como la psicológica. Este artículo se centra en el aspecto psicológico de la supervivencia. 


\section{ASPECTOS PSICOLÓGICOS A CONSIDERAR EN UNA SITUACIÓN DE SUPERVIVENCIA}

Hay una regla básica en supervivencia que marca las limitaciones físicas del ser humano ${ }^{6}$ :

a) «El ser humano es capaz de sobrevivir hasta tres semanas sin comida».

b) «El ser humano es capaz de sobrevivir hasta tres días sin agua».

c) «El ser humano es capaz de sobrevivir hasta tres minutos sin aire».

A esta regla podemos añadirle una premisa más: «El ser humano puede perder la vida en tres segundos si no es capaz de mantener la calma». Efectivamente, en toda situación de supervivencia hay que considerar la existencia de ciertos factores relacionados con el estado psicológico del superviviente que condicionan la forma en que se enfrenta a esa situación. Nos referimos a aspectos como la ansiedad y el miedo, la culpa, la irritabilidad, la soledad o la depresión. Por otra parte, otros condicionantes de tipo fisiológico como la sed, el cansancio o el dolor poseen un fuerte componente vivencial o subjetivo, de modo que la forma en que el individuo los experimenta o vive se ve muy influida por su estado psicológico.

Siguiendo un esquema tradicional ${ }^{3}$, a continuación se exponen una serie de aspectos psicológicos que condicionan la actitud y comportamiento del superviviente:

\section{Soledad}

El hombre es un ser social, acostumbrado a vivir rodeado de sus semejantes; pocas personas sienten la necesidad de vivir solos. Además, vivimos en una sociedad que proporciona pocas oportunidades de poner a prueba nuestra habilidad para adaptarnos a la soledad, a la ausencia de los demás y a la falta de apoyo social ${ }^{7}$. Por otra parte, la formación e instrucción militar fomenta en buena medida la cohesión y el trabajo en equipo, es decir, la interacción e interdependencia con los demás. Sin embargo, en una situación de supervivencia existen muchas posibilidades de acabar aislado física o psicológicamente, sin posibilidad de recurrir a la ayuda de otros. En este último caso nos referimos a la llamada soledad emocional, que se produce cuando pese a encontrarse en un grupo el superviviente se siente aislado de los demás ${ }^{1}$. Esta soledad es más perjudicial desde el punto de vista psicológico, ya que además de los problemas que origina en el sujeto que se aísla, tiene repercusiones negativas en el grupo (negativismo, problemas de relación e interacción, y menor cohesión, etc.).

Por tanto, es fundamental para todo militar adquirir durante su entrenamiento un elevado grado de autosuficiencia, que le permita hacer frente a situaciones caracterizadas por ese aislamiento y en las que deberá desenvolverse por sí mismo.

\section{Culpabilidad}

Las circunstancias que llevan a una persona a una situación de supervivencia pueden resultar trágicas y dramáticas, como ocurre en caso de accidentes o misiones que implican bajas amigas ${ }^{3}$. En estos casos no es raro que el superviviente, al tiempo que se alegra por permanecer vivo, se sienta afligido por la muerte de sus compañeros, apareciendo entonces sentimientos ambivalentes: por un lado sentirse afortunado por haber sobrevivido, por otro experimentar un sentimiento de culpa por no haber sufrido el mismo destino que sus compañeros.

El superviviente debe aprender a manejar la culpabilidad de forma positiva, y no permitir que le arrebate las ganas de vivir. No hay nada más absurdo que rechazar la posibilidad de sobrevivir, más aún cuando otros compañeros no lo consiguieron.

\section{Ansiedad y miedo}

Experimentar miedo y ansiedad es una reacción normal que se relaciona con el instinto de conservación y que previene al ser humano contra las situaciones que valora como amenazadoras para su integridad. En una situación de supervivencia podemos sentir ansiedad y miedo ante muchos estímulos: lo desconocido, perdernos, morir, el dolor, nuestras propias debilidades, etc. Empleados de manera efectiva y controlada, estos sentimientos pueden impulsar a la persona a enfrentarse a los peligros que le amenazan, estimulándole a luchar por su supervivencia. El miedo agudiza los sentidos, nos mantiene alerta y nos prepara para la lucha. No obstante, cuando el superviviente es incapaz de controlar la ansiedad y el miedo que experimenta, estas reacciones se convierten y manifiestan en conductas inadecuadas y desadaptativas (irritabilidad, agresividad, crisis de pánico o angustia) que pueden impedirle desarrollar las actividades necesarias para su supervivencia.

La ansiedad presenta un triple componente -fisiológico, cognitivo y conductual- que todo militar debe conocer y que se resumen en la Tabla 1 .

En una situación de supervivencia, estas manifestaciones pueden ser desencadenadas por determinados estresores que el militar debe conocer para poder identificarlos, anticiparse a ellos y desarrollar estrategias para afrontarlos. Aspectos fundamentales en supervivencia como la preparación y el entrenamiento, las experiencias previas, la actitud personal ante la vida y el nivel de autoestima y autoconfianza van a mediar en la percepción del superviviente acerca de qué factores son estresantes para él, y cuáles no.

Entre estos desencadenantes de la ansiedad y el miedo podemos señalar ${ }^{3,8}$ :

a) Dolor, enfermedad y muerte. Estar herido o enfermo puede generar ansiedad en el superviviente, al limitar su capacidad para realizar determinadas actividades necesarias para subsistir, como encontrar refugio, obtener recursos, desplazarse, o escapar y defenderse del enemigo. Por otra parte, no hay nada más paralizante e incapacitante para el superviviente que el miedo a morir.

b) Incertidumbre y falta de control. En toda situación de supervivencia predomina la incertidumbre, causada por la ausencia de información (¿dónde estoy?, ¿qué va a pasar?, ¿me estarán buscando? o ¿cuando me rescatarán?, etc.). Por otra parte, determinados individuos sienten la necesidad de poseer el control de todo lo que le ocurre y rodea, de modo que la falta de control propia de toda situación de supervivencia supone una fuente de estrés para ellos 5 .

c) Medio ambiente. Nos referimos a estresores externos como el miedo a lo desconocido, los agentes atmosféricos (calor, frío, nieve, lluvia, viento intenso), el terreno (montañas, pantanos o cenagales, 
Tabla 1. Principales manifestaciones de la ansiedad (adaptado del modelo tridimensional de la ansiedad de Lang ${ }^{16}$ ).

\begin{tabular}{|lll|}
\hline Manifestaciones fisiológicas & - Aumento del ritmo respiratorio & - Espasmos, temblores \\
& - Aumento de tasa y presión cardíaca & - Sudoración excesiva \\
& - Palpitaciones, presión o dolor en pecho & - Sequedad de boca \\
& & - Pérdida de apetito \\
& - Nauseas, vómitos y diarrea & - Fatiga / cansancio extremo \\
\hline Manifestaciones cognitivas & - Alteración de la atención, concentración y memoria & - Preocupación excesiva \\
& - Indecisión & - Pérdida de autoconfianza \\
& - Insomnio, pesadillas, terrores nocturnos, etc.) & - Labilidad emocional \\
& - Hesesperanza & - Altersensibilidad al ruido, moves de la percepción \\
& - Hipervigiento y luz \\
\hline Manifestaciones conductuales & - Apatía y aislamiento social \\
& - Irritabilidad y agresividad & - Apariencia ausente \\
& - Conducta de evitación y/o huida & - Ataques de pánico \\
& - Dificultad para comunicarse & \\
\hline
\end{tabular}

desiertos) o la fauna que habita en la zona en que se encuentre el superviviente (animales salvajes, insectos y reptiles peligrosos).

d) Carencia de agua y alimentos, o la dificultad para conseguirlos, supone una importante fuente de estrés.

e) Fatiga. Una situación de supervivencia puede prolongarse durante días o semanas, exigiendo al superviviente realizar esfuerzos intensos y prolongados que pueden causarle cansancio y agotamiento. La fatiga se traduce en una disminución de las capacidades físicas (con enlentecimiento motor, dificultades de coordinación, etc.) y cognitivas (disminución de la atención y estado de alerta, aumento del tiempo de respuesta y de la tasa de errores, etc.) esenciales para la supervivencia.

f) Aislamiento. Un desencadenante importante del estrés es que a menudo el superviviente se encuentra solo y debe confiar únicamente en sus propios recursos para sobrevivir.

\section{Frustración}

Cuando una persona fracasa continuamente en sus intentos de lograr determinada meta corre el riesgo de caer en la impotencia y frustración. Para permanecer vivo hasta conseguir ayuda, el superviviente debe realizar determinadas tareas con escasos medios, por lo que resulta inevitable que cometa errores o que algo salga mal o escape a su control ${ }^{3}$. Situaciones frustrantes en supervivencia son las dificultades para encontrar recursos básicos (alimentos y agua), perderse, condiciones climatológicas desfavorables (calor extremo, lluvia torrencial), el terreno inhóspito, la presencia de patrullas enemigas o las limitaciones físicas provocadas por heridas, cansancio o agotamiento. Tarde o temprano, el superviviente deberá enfrentarse con la frustración y con las consecuencias propias de ese estado, como la irritabilidad, la impulsividad, la agresividad, los comportamientos irracionales y la desesperanza.

\section{Depresión}

En una situación de supervivencia es frecuente experimentar sentimientos de tristeza o desesperanza. Estos sentimientos no tienen porqué ser negativos, e incluso pueden impulsar nuestro deseo de sobrevivir con más fuerza. En cambio, cuando se agra- van o se prolongan en el tiempo pueden desembocar en estados de depresión.

Como es bien sabido en Psicología, la depresión se relaciona estrechamente con la frustración y la ira. La persona frustrada se vuelve cada vez más irritable conforme fracasan sus intentos de alcanzar sus metas, con lo que su frustración aumenta cada vez más ${ }^{3}$, estableciéndose así un círculo vicioso que se autoalimenta hasta que el superviviente se derrumba física, mental y emocionalmente. Llegado a este punto, pueden aparecen conductas autolíticas y pensamientos depresivos del tipo «este es el final», «no merece la pena seguir viviendo» o «no hay nada que pueda hacer».

\section{MEDIDAS PSICOLÓGICAS PARA AFRONTAR UNA SITUACIÓN DE SUPERVIVENCIA}

Una vez analizados los aspectos anteriores, se proponen una serie de pautas y medidas psicológicas que nos ayudarán a afrontar una situación de supervivencia.

\section{Tener siempre presente la posibilidad de verse envuelto en una situación de emergencia}

Ningún militar desea verse envuelto en situaciones peligrosas o de supervivencia, pero estas situaciones tienen un fuerte componente de sorpresa y se caracterizan por lo inesperado. No se trata de ponerse siempre en lo peor, ni en dejar que la posibilidad de verse envuelto en una situación de emergencia domine nuestra vida o actuación profesional. Pero debemos ser conscientes de la probabilidad de que nos ocurra, sobre todo en determinados momentos y situaciones como maniobras, misiones en el exterior o desplazamientos sobre terrenos desconocidos o inhóspitos.

\section{Conocerse a sí mismo}

Aunque sorprendente, la capacidad del ser humano de adaptarse a las nuevas situaciones y circunstancias cambiantes de su entorno es limitada. Es importante para todo militar conocer cuál es su capacidad de adaptación y evaluar sus aptitudes para la supervivencia, 
lo que le permitirá detectar sus puntos débiles y desarrollar aquellas habilidades necesarias para sobrevivir ${ }^{3}$. Por otra parte, a lo largo de nuestra vida hemos tenido que enfrentarnos con situaciones que han puesto a prueba nuestra resistencia y límites psicológicos: grandes esfuerzos físicos, pérdida de personas queridas, rupturas sentimentales... Debemos analizar estas situaciones objetivamente, ser consciente de qué sentimos, cómo nos afectaron y reaccionamos ante ellas, para aprender de la experiencia y mejorar nuestra resistencia psicológica.

\section{Estar preparados}

Una adecuada preparación y entrenamiento en supervivencia es positiva para todo militar, ya que fortalece el cuerpo y la mente, aumenta su autoestima y autoconfianza, fomenta la capacidad de improvisación y le prepara para afrontar con éxito una situación de supervivencia real. Entrenamiento y preparación marcan la diferencia entre sobrevivir y morir 9 . Aunque en relación con otras especies animales, los instintos humanos se encuentran atrofiados, el hombre cuenta con un arma única: su capacidad para aprender racionalmente una serie de conductas y habilidades que podrá ejecutar posteriormente cuando las necesite. Aplicada a la supervivencia, esta preparación abarca tres aspectos fundamentales: el técnico, el físico y el psicológico $^{2}$ :

a) Conocimientos teóricos y técnicos. La adquisición de estos conocimientos sobre supervivencia y el entrenamiento lo más realista posible (ejercicios y prácticas de supervivencia), permitirán al militar aprovechar los recursos naturales y suplir con ellos la falta de equipo adecuado ${ }^{2}$. Podemos mencionar el manejo de planos y brújulas, la orientación nocturna, la construcción de refugios, primeros auxilios, artes de caza y pesca, etc.

b) Forma física. Poseer una buena forma física es un factor que confiere mayor probabilidad de sobrevivir ${ }^{10}$. Sin embargo, lo peor que le puede ocurrir al superviviente no es encontrarse en baja forma, sino desconocer sus capacidades físicas y su nivel de resistencia reales. Por ello, todo militar debe valorar su fuerza y resistencia de forma objetiva y realista, sin dejarse influir por marcas o experiencias pasadas. Una vez inmerso en la situación de supervivencia, las tareas y actividades que realicemos deberán ser acordes a nuestras capacidades y preparación física, para no malgastar energías inútilmente.

c) Entrenamiento psicológico. Hemos visto que el superviviente va a experimentar una serie de reacciones que pueden incapacitarle o derrotarle en caso de no ser controladas adecuadamente: miedo, ansiedad, irritabilidad, etc. Pero utilizados adecuadamente, estos sentimientos y reacciones incrementan sus posibilidades de sobrevivir, haciéndole más fuerte y seguro de sí mismo.

\section{Adoptar una actitud adecuada y positiva: voluntad de sobrevivir}

La voluntad de resistir y sobrevivir es posiblemente el factor más importante de la supervivencia ${ }^{2,7,11,12}$. La historia está repleta de ejemplos que muestran que la mente y la voluntad tienen la capacidad de exigirle al cuerpo más de lo que éste puede resistir. A menudo, la fatiga que sufre un superviviente es una falsa señal que no se debe al agotamiento real de su capacidad de resistencia, sino a factores psicológicos como la desesperanza o la frustración que pueden llevarle al abandono y a la derrota. Es fundamental, por tanto, poseer una actitud psicológica fuerte que nos permita enfrentarnos sin desfallecer a la desesperación, la angustia, el tedio, el dolor, el hambre o la fatiga; sensaciones y sentimientos éstos que, como dijimos anteriormente, poseen un fuerte componente subjetivo. Esta actitud se caracteriza por:

a) Una moral elevada. Desde el punto de vista militar podemos definir moral como un estado de ánimo positivo hacia el cumplimiento de la misión. En una situación de supervivencia, nuestra misión es sobrevivir conservando la capacidad operativa, por lo que mantener la moral elevada contribuirá a aumentar las probabilidades de supervivencia.

b) El buen humor. Reírse de uno mismo y de sus circunstancias, aún en condiciones adversas, es una actitud saludable que influye favorablemente en la supervivencia ${ }^{13}$.

c) El optimismo. Es importante ver el lado positivo de las cosas, ya que de todas las experiencias -incluso de las más adversas- se puede aprender. Con la actitud adecuada, una situación de supervivencia puede convertirse en una experiencia enriquecedora, una oportunidad única para explorar nuevas áreas y conocernos mejor. En cambio, una actitud negativa, caracterizada por el pesimismo, el abatimiento o la derrota conlleva una disminución de la moral y motivación, con lo que se reducen también las probabilidades de sobrevivir.

\section{Saber reorganizarse y elaborar un plan de acción}

Llegado el momento, es fundamental conservar la calma y recordar los conocimientos y técnicas aprendidas durante el entrenamiento en supervivencia. Para ello es recomendable elaborar un plan de acción, que incluya los siguientes pasos:

1. ${ }^{\circ}$ No precipitarse. En una situación de supervivencia es fundamental conservar las fuerzas y energías, no malgastándolas en actividades poco útiles para el objetivo de sobrevivir. En este sentido, descanso, sueño y calma son buenos aliados para el superviviente.

2. Evaluar la situación de manera realista, prestando especial atención a aspectos como la presencia de heridos y de peligros o amenazas para nuestra integridad. También hay que considerar las posibilidades de desplazarnos a zonas más adecuadas o propicias para sobrevivir y obtener recursos como agua, alimentos o fuego. El análisis de la situación debe ser lo más realista y objetiva posible, ya que mantener unas expectativas irreales puede conducirnos al desánimo ante el primer contratiempo que surja.

3. ${ }^{\circ}$ Establecer metas intermedias que nos acerquen al objetivo final de sobrevivir. Establecer estas metas y centrarnos en alcanzarlas estimula nuestra creatividad, nos motiva y nos mantiene vivos. Entre estas metas intermedias están colocar marcas o señales que faciliten el rescate, buscar o construir un refugio donde resguardarse, o conseguir agua, alimentos u otros recursos que pudiéramos necesitar.

4. ${ }^{\circ}$ Realizar un mapa mental de la zona en la que nos encontremos, atendiendo a la topografía y fisiografía del lugar y a la presencia de puntos característicos en el terreno como accidentes geográficos (collados, cumbres, valles), el tipo de vegetación, 


\section{Aspectos psicológicos de la supervivencia en operaciones militares}

construcciones o elementos artificiales (excavaciones, sendas, pistas), relieve (curvas de nivel), presencia de elementos característicos (árboles derribados, rocas con formas curiosas), etc. Ello será de gran utilidad si hemos de alejarnos del lugar donde hayamos instalado el campamento para explorar el entorno o buscar agua o alimentos.

\section{Controlar el miedo}

La supervivencia depende en gran medida de la habilidad para manejar adecuadamente el estrés propio de la situación. En consecuencia, todo militar debe entrenarse para autocontrolar sus miedos, para percibirlos como algo natural y útil, y poder desenvolverse bajo sus efectos. Algunas medidas efectivas para ello son:

a) Fomentar una preparación adecuada y un entrenamiento realista en supervivencia, que permita adquirir los conocimientos y habilidades necesarios para aumentar nuestra confianza y seguridad en nosotros mismos.

b) Aceptar que el miedo es una reacción normal y positiva para la supervivencia, por lo que es importante aprender a reconocer los propios miedos y ser consciente de cómo pueden afectarnos en esa situación.

c) Confiar en sí mismo y en las propias posibilidades ${ }^{7}$. A menudo, en supervivencia habremos de confiar exclusivamente en nosotros mismos, en nuestros propios recursos, capacidades y habilidades para sobreponerse a las adversidades y sobrevivir.

d) Mantenerse constantemente atentos a lo que ocurre alrededor, para anticipar prevenir y detectar la existencia de posibles amenazas, riesgos y peligros.

e) Aplicar la sensatez y no dejar que la imaginación nos juegue malas pasadas. Muchos de nuestros miedos son tan infundados que no resisten al análisis de la lógica. Así, los animales salvajes rara vez atacan al hombre, más bien los evitan; y la mayoría de ruidos nocturnos, por muy amenazantes y peligrosos que nos puedan parecer, son solo ruidos.

f) Mantener el buen humor es un buen antídoto contra el estrés, la ansiedad y el miedo.

g) Por último, existen técnicas psicológicas de probada eficacia en el control de la ansiedad, como la relajación, detención del pensamiento, autoinstrucciones e inoculación de estrés.

\section{Adaptarse a la nueva situación}

La creatividad, la capacidad de improvisación y adaptación son aspectos claves en toda situación de supervivencia ${ }^{1,14}$. En estas situaciones, caracterizadas generalmente por un medio desconocido y hostil en el que las posibilidades de acceder a recursos están limitadas, deberemos usar nuestra imaginación y habilidad de improvisar para adaptarnos con éxito ${ }^{7}$.

Por otra parte, no hay dos situaciones de supervivencia iguales. No es lo mismo intentar sobrevivir en el desierto que hacerlo en un bosque tropical, ni todo se puede aprender en cursos o manuales. Generalmente, aprendemos unas habilidades básicas que después habremos de poner en práctica en la situación de supervivencia específica en función de sus peculiaridades y características. Es decir, habremos de introducir modificaciones sobre los conocimientos ad- quiridos, adaptándolos a la situación, lo que requiere grandes dosis de flexibilidad, creatividad y facilidad para improvisar y solucionar problemas.

\section{Mantenerse ocupado}

Dado que somos seres sociales, acostumbrados a relacionarnos y desenvolvernos en grupos, el aislamiento, la soledad y el aburrimiento merman nuestras posibilidades y deseos de sobrevivir, conduciéndonos a la desesperanza. Para evitarlo, es fundamental tener ocupada la mente en actividades y tareas que aumenten nuestras probabilidades de sobrevivir: cuidar la higiene personal, recolectar alimentos, preparar señales para ser rescatado o mejorar la habitabilidad del refugio ${ }^{15}$. Otras actividades para mantenerse ocupado pueden ser elaborar un plan de actividades que nos imponga disciplina al cuerpo y la mente (asearse, mantenerse en forma, practicar relajación, o buscar comida, etc.) o llevar un diario en el que anotar datos importantes de lo que nos acontece. En definitiva, se trata de evitar por todos los medios caer en la desesperación, realizando cualquier tarea que nos mantenga distraído u ocupado.

\section{CONCLUSIONES}

Aprender supervivencia es un requisito fundamental para todo militar, ya que a lo largo de su vida profesional se va a ver implicado en una serie de misiones y operaciones que pueden desembocar en una situación de supervivencia: participar en misiones de paz o interposición de fuerzas, maniobras, ejercicios militares, enfrentarse con desastres naturales.

La mayor parte del adiestramiento y entrenamiento militar en supervivencia se centra en la preparación física y en la adquisición y desarrollo de una serie de habilidades o destrezas técnicas necesarias para sobrevivir, como construcción de refugios, obtención de alimentos, orientación, etc. Dicho entrenamiento apenas tiene en consideración diversos aspectos psicológicos cuya presencia es inherente a toda situación de supervivencia, como la ansiedad y el miedo, la soledad o la desesperanza. Sin embargo, la experiencia ha demostrado la importancia de estos aspectos, que a menudo limitan y dificultan la adaptación del superviviente, convirtiéndose en poderosos enemigos de la supervivencia. Por ello, todo militar debe conocer estos aspectos y ser consciente de su importancia para poder identificarlos, prevenirlos y saber enfrentarse a ellos. A este respecto se ha propuesto una serie de medidas y pautas de conducta cuyo objetivo es aumentar las posibilidades de supervivencia. Estas medidas giran en torno a la necesidad de que, dada la naturaleza de las misiones y operaciones que le pueden ser encomendadas, el militar sea conciencie de la posibilidad de verse envuelto en estas situaciones y se conozca a sí mismo, sus límites físicos y psicológicos. Este es el primer requisito de un adecuado entrenamiento en supervivencia, entrenamiento que debe incluir también una preparación física, técnica y psicológica, que posibilite al militar afrontar una situación de supervivencia. Para ello deberá poner en marcha un conjunto de estrategias y conductas que, impulsadas por una actitud positiva y un fuerte deseo de sobrevivir, le permita superar la adversidad y adaptarse a la nueva situación. 
En consecuencia, el entrenamiento militar en supervivencia debería otorgar mayor relevancia a la influencia de las variables psicológicas y a la preparación psicológica del combatiente. A ello puede contribuir en buena medida la Psicología Militar, no solo en la vertiente docente y asistencial, sino también en el fomento y elaboración de estudios e investigaciones sobre la influencia de los aspectos psicológicos en las situaciones de supervivencia, la vulnerabilidad psicológica ante estas situaciones, o la importancia de los estilos de afrontamiento, etc.

\section{BIBLIOGRAFÍA}

1. Rangel, C. Supervivencia: aspectos psicológicos [en línea] [acceso 11 de junio de 2009]. Disponible en URL: http://montanismo.org.mx/articulos.php?id sec=7\&id art=284

2. Estado Mayor del Ejército. ME6-003. Manual de Enseñanza. Supervivencia Tomo 1. Madrid: Ministerio de Defensa, 1992: 1-1 a 1-16.

3. Headquarters Department of the Army. U.S. Army Survival Manual, FM 21-76. Chap. 2. Washington, DC, 1992: 8-13.

4. Estado Mayor del Ejército. M17-002. Manual de Instrucción. Supervivencia. Madrid: Ministerio de Defensa, 1994.

5. Motley, E.B. Survival Stressors faced by Military Aviators/Aircrew Following Ditching Over Salt Water. En 43rd Annual Symposium Papers. Salt Lake City, Utah. Oregon: SAFE Association, 2005: 227-241.
6. Palma, A. Sobrevivir. Guía y Técnicas de Supervivencia [en línea] [acceso 8 de julio de 2009]. Disponible en URL: http://www2.udec.cl/ lpalma/supervivencia.html

7. Conway, C. The attitude of survival [en línea] [acceso 18 de junio de 2009]. Disponible en URL: http://www.geocities.com/Yosemite/Falls/9200/survival_attitude.html

8. U.S. Marine Corps. MCRP 3-02H. Survival, Evasion and Recovery. Quantico, Virginia: USMC, 1999

9. Ferrero, F. La necesidad de la motivación para la supervivencia (I) [en línea] [acceso 23 de junio de 2009]. Disponible en URL: http://www.andinia.com/articles/ supervivencia sobrevivir/a23787.shtml

10. Wiseman, J. Manual de Supervivencia del SAS (Special Air Service). Barcelona: Editorial Acanto, 1986: 9.

11. Escuela Argentina de Supervivencia. Psicología de la supervivencia [en línea] [acceso 13 de julio de 2009]. Disponible en URL: http://www.supervivenciaeas. com.ar/

12. Beeson, C. Manual de supervivencia en el mar. Madrid: Editorial Libsa, 2004: 45

13. Centro de Psicología de la Flota. Guía para la supervivencia psicológica. Base Naval de Rota.

14. Rousseau, M. Psychologie de la Survie [en línea] [acceso 1 de julio de 2009]. Disponible en URL: http://www.serabec.org/formation/psychologie_de_la_survie.pdf

15. Cuerpo de Socorro Andino. Manual de supervivencia: 2-4 [en línea] [acceso 14 de julio de 2009]. Disponible en URL: http://www.andinismogea.cl/manual_supervivencia.pdf

16. Lang, Peter J. Fear reduction and fear behavior: Problems in treating a construct En Shlien, J.M (Ed.). Research in psychotherapy, vol I. Washington, DC: American Psychological Association, 1968: 90-102. 